\title{
Design and Optimized Simulation of Seedling Tray Stacking Manipulator for Seedling Machine Producing Square Substrate Blocks
}

\author{
Tianhua $\mathrm{Li}^{1,4}$, Guangwei Liu ${ }^{2}$, Yuhua $\mathrm{Li}^{1}$, Yueshun $\mathrm{Zhang}^{1}$, Jialin $\mathrm{Hou}^{1}$, Guoying Shi ${ }^{1,3, *}$ and \\ Kai Zhou ${ }^{1}$
}

\author{
${ }^{l}$ College of Mechanical and Electronic Engineering, Shandong Agricultural University, Tai'an 271018, China \\ ${ }^{2}$ Taian Institute of Supervision \& Inspection on Product Quality, Tai'an 271018, China \\ ${ }^{3}$ Shandong Provincial Engineering Laboratory of Agricultural Equipment Intelligence, Tai'an 271018, China \\ ${ }^{4}$ Precision and Automated Agriculture Lab, University of Missouri, MO 65211, USA
}

Received 3 August 2019; Accepted 20 October 2019

\begin{abstract}
Industrialized seedling has the advantages of high efficiency, low cost, easy promotion of fine varieties, and standardized seedling management, so it is the trend of modern agriculture development. Seedlings using square substrate blocks is one of the main ways, but the high labor intensity and high labor costs have hindered its rapid development. In order to realize the automatic unloading of the seedling trays, this study designed and optimized a kind of seedling tray stacking manipulator for nursery machine that produces square substrate blocks. A design method for the robustness of grabbing the seedling trays and the anti-interference between manipulator and seedling tray was also proposed. Firstly, the principle of stacking manipulator was analyzed, and the structure was optimized. Kinematics analysis was conducted, and a kinematic model was established. The relationship between the stroke of an electric push rod and the opening and closing angle of a claw was obtained through model analysis. The movement stroke of the electric push rod was optimized to meet the design requirements of the maximum and minimum limit positions of the manipulator. Finally, the rationality of the parameter design was verified by static and kinematics simulation. The results demonstrate that the stroke range of the electric push rod is $27.6-105.6 \mathrm{~mm}$, and the opening and closing angle range of the claw is $11.7-66.7^{\circ}$, which meets the requirements. In addition, the force of each component during the seedling moving is within a reasonable range. This study provides an important reference for the development and performance improvement of automatic square substrate seedling machines that support automatic loading, unloading, and palletizing.
\end{abstract}

Keywords: Seedling machine, Stacking manipulator, Seedling tray, Parameter optimization, Simulation analysis

\section{Introduction}

Facility agriculture has developed rapidly with the adjustment of structures for agricultural industry and planting. Vegetable cultivation area has expanded, and largescale planting has gradually become the trend of modern agricultural development. However, the traditional way of growing seedlings is labor-intensive; hence, its reformation and the implementation factory seedlings are an inevitable choice to improve the production of vegetables and cash crops in China [1]. The main advantages of factory seedlings are labor-saving, low cost and high efficiency. This method is convenient for the promotion of fine varieties and standardized seedling management. After rooting, the roots have good vitality, and seedlings grow fast, which is conducive to the cultivation of robust seedlings and effectively reduces the spread of pests and diseases. Mechanization, industrialization, and commercialization of vegetable seedling and production [2] can be realized. The seedling machine is the core equipment for factory seedling production and is roughly divided into two categories according to the operation process: plug seedling raising machine and square substrate seedling machine [3].

*E-mail address: 13518683945@163.com

ISSN: $1791-2377$ @ 2019 School of Science, IHU. All rights reserved. doi:10.25103/iestr.125.18
Square substrate seedling is one of the main ways of planting seedlings. This method has the advantages of high efficiency and low cost and is the development trend of mechanized seedlings [4]. However, its rapid development has been hindered due to the high labor intensity and high labor cost of the seedling tray's handling. Therefore, developing an efficient and reliable automatic seedling tray palletizing device for square substrate seedling machine is of great importance.

Plug seedling raising machine in other countries has a research history of nearly 50 years; hence, the equipment and technologies are perfect and mature [5-9]. The main instruments used are Blackmore, Speeding, EZ, Vandana in the United States, Hamilton in the United Kingdom, Visser in the Netherlands, Willimeses in Australia, Helper seeders in Korea's Dadong Electromechanical, and Yanmar and Kubota in Japan $[10,11]$. By contrast, research on the seedling raising device in China is relatively late. The seedling raising technology was introduced in the 1980s [12]. The equipment developed by universities and research institutes mainly consists of 2XB-400 plug seedling precision seeding machine, which was jointly developed by China Industrial Engineering Research and Design Institute and China Agricultural University, and 2QB-330 air suction vibrating seedling precision seeding machine, which was jointly developed by Nanjing Agricultural Mechanization 
Research Institute and Jiangsu University. 2ZBQ-300 double-layer drum air suction seeding machine was developed by Guangxi Agricultural Mechanization Research Institute, and 2BJOP-120 and SZ-200 seeding machines were developed by Beijing Agricultural Machinery Research Institute [13].

Only a few manufacturers produce square substrate seedling machines in China as compared with tray seeders. The mature products are the $2 z b-100$ produced by Zhongnongji Fengmei and the 2zb-60 by Shandong Huaxing Machinery Co., Ltd. The seedling tray used by the square substrate seedling machine has no holes, the handles are provided at both ends of the seedling tray, and the substrate is directly diced in the seedling tray. The substrate used for filling, particularly a light substrate such as peat, must be added to the soil. Water is added before the filling. Therefore, the density of the substrate used is relatively large, and the seedling tray after the filling of the substrate is heavy. Unloading the tray and transporting it to the designated location from the machine's conveyor increase the labor intensity and cost. On the contrary, the mechanization degree of the whole seedling operation is also reduced.

Basing on the above analysis, this study uses the planar link structure to optimize the design of a palletizing robot and to improve the automation degree of square substrate seedling machine. Manual operation is replaced to realize the fast and accurate palletizing of the seedling tray. The key parameters of the palletizing robot movement model are optimized to improve its working efficiency and reliability. This work provides a useful reference for further study on automatic palletizing for seedling machines.

\section{State of the Art}

Many scholars studied the fully automated production of facility nursery machinery. Chiu et al. [14, 15] developed a generator-powered, self-propelled automatic tray loading/unloading machine for rice seedling nurseries by using a gantry as the transport system to automate seedling tray placement and harvesting. This equipment enables the placement of seedling trays from conveyor belts to seedbeds and vice-versa to the automatic seedling tray stacking. However, its seedling lifting and transferring mechanism cannot meet the structural requirements for the seedling tray of square substrate seedling machine. Xiao et al. [16] developed a combined seedling tray automatic delivery system for automated transplanting. Owing to the control based on conveyor belt movement, the requirements for trays loading automation cannot be realized. Jin et al. [17] designed the vegetable seedling automatic conveying system to improve the automation and reliability of mechanized transplanting. However, the special supporting storage mechanism required for the seedling tray transmission does not satisfy the existing supporting equipment of the square substrate seedling tray conveying system. Rosli et al. [18] developed seeding tray placement and transfer devices for rice seeding systems to increase seeding efficiency, but the loading and unloading trays are still manually completed. Han et al. [19] developed a door-frame pendulum device for automatic field transplanter. This equipment can simultaneously extract two seedlings from the tray and transfer them to the transplant discharge point. The structure and principle have guiding importance to the seedling tray's transfer and stacking, but the device itself cannot achieve seedling loading/unloading automation. Choi et al. [20] developed a new picking device for vegetable transplanters; this instrument extracts seedlings from trays and transfers them to a transplantable position, but it could not be used for the transfer and stacking of seedling trays. Ma et al. [21, 22] designed an equipment for the automatic stacking of hard and soft sputum in the precision seeding line of rice cultivation and a pneumatic automatic feeding device for the rice cultivating seedling machine; both met the requirements for common soft and hard smashing plates, stacking requirements, and automatic feeding. However, the two devices can only be applied to a specific seedling tray and can only complete the tray feeding operation, thus unable to satisfy the requirements of the grab-type seed tray shifting and stacking and realize shift and stack. Quan et al.[23] proposed a seedling handling robot in a logistics and confined space inside a plant factory. This machine fulfilled the required handling functions in the plant and relied on the robotic arm to achieve a specific level of handling. The square substrate seedling tray's torque and structural requirements cannot be satisfied in this way. Li et al.[24] established a dynamic model for the lateral transfer drive crankshaft-double rocker-gear transmission of dryland transplanter seedling tray and analyzed the connection point between crank, connecting rod, rocker, and gear. The force with the meshing point changes with the crank angle, but the possible interference was not analyzed. Wei et al. [25] designed and develop a prototype for a tobacco planter. The seedling tray drive device ensured that it could travel at a certain speed, but the seedling plate still needs to be manually unloaded after the pressure hole is completed. Ren et al. [26] developed a seedling tray automatic feeding system, which realizes the automatic, individual feeding of seedlings by controlling the horizontal and vertical intermittent movement of the seedling tray. Jin et al. [27] designed a new automatic seedling transplanting device that consists of a vertical movement mechanism, ejecting mechanism, horizontal movement mechanism, guiding and gripping mechanisms. The device meets the requirements of the lateral shifting plate and the longitudinal stepping shifting plate, but the movement and stacking of the entire seedling tray as a whole cannot be realized. Chiba et al. [28] proposed a working environment optimization design method to improve the performance of the palletizing robot. The environmental parameters can be quickly evaluated according to the basic position of the robot and the shape and position of the pallet. In addition, the calculation time of the path planning is reduced, but the possible interference in the robot movement was not analyzed. Nakamoto et al. [29] designed a high-speed and compact depalletizing robot using deep image processing to identify the processing sequence of the package with gantry robots and telescopic arm mechanisms. However, the telescopic arm structure is not suitable for seedling machine transport mechanisms, and the processing order cannot meet the requirements of the stacking trays. Nguyen et al. [30] proposed an optimization algorithm for determining the optimal operating room layout and a motion trajectory for planning the end actuator of the robot with optimized average deviation. Kinematic standards and optical constraints were used for surgically assisted robotic systems, but the amplitude of the end actuator per step cannot be determined.

The above studies mainly focused on different aspects of plug seedling automation, from seedling tray to seedbed, seedling tray to conveyor belt, and the stepping movement control of seedling tray in seedling and transplanting. In view of the big size of square substrate seedling tray, large 
substrate density and unique structure, the study on seedling tray loading/unloading and stacking is relatively few. Only a limited number of interference analyses is available in the study of the end actuator of the manipulator, and no accurate amount of hand opening, closing, and movement has been established. In the present study, the transmission structure and seedling tray characteristics of the square substrate seedling machine are combined with the manual unloading and palletizing action. An unloading palletizing robot is proposed, and the kinematic model is established to determine the interference between the robot and the seedling tray during work. The performance of the manipulator is optimized and guaranteed for the long-term stable operation of the manipulator.

The remainder of this study is organized as follows. Section 3 establishes the structure and working principle of the unloading palletizing device and the manipulator, and kinematics and static analyses of the manipulator are conducted. Section 4 discusses the 3D model of the palletizing robot in SolidWorks and Adams. Simulation is performed with the claws at the minimum and maximum limit positions. The relationship between the simulated values and the theoretical values is obtained. Section 5 summarizes the study and draws the conclusions.

\section{Methodology}

\subsection{Structure and working principle}

The unloading palletizing device shown in Fig. 1 mainly includes a frame, a palletizing robot, XYZ-direction slides, a synchronization rod, a coupling, and two servo motors. The palletizing robot is installed on the slider of the z-direction slide, and its structure is shown in Fig. 2 is composed of a vertical fixing rod, a horizontal fixing rod, an electric push rod, a connecting rod, and a claw.

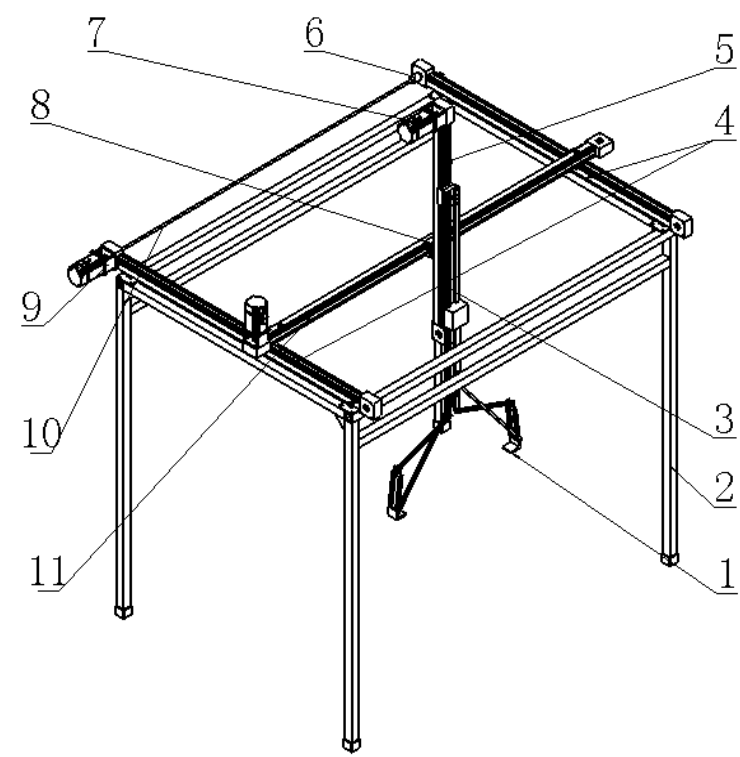

1. Palletizing manipulator 2. Frame 3. Slide-way of the $Z$ direction 4 . Slide-way of the $X$ direction 5. Limit switch 6. Coupling 7. Servo motor 8. Slider of the $Y$ direction 9. Motor holder 10. Synchronous rod 11. Slide-way of the $Y$ direction

Fig. 1 Unloading palletizing device

During operation, the servo motor drives the slider to run along the slideway to the top of the seedling tray conveyor belt. The electric push rod opens the claw that will be grabbed by the seedling tray and subsequently drives the gripper to close and grasp the seedling tray. The handle of the palletizing robot and the seedling tray move along the slide with the slider to the designated position. Finally, the electric push rod drives the gripper to open completely and away from the seedling tray. The palletizing of a seedling tray is completed, and the continuously opening gripper is ready to grab the next seedling tray.

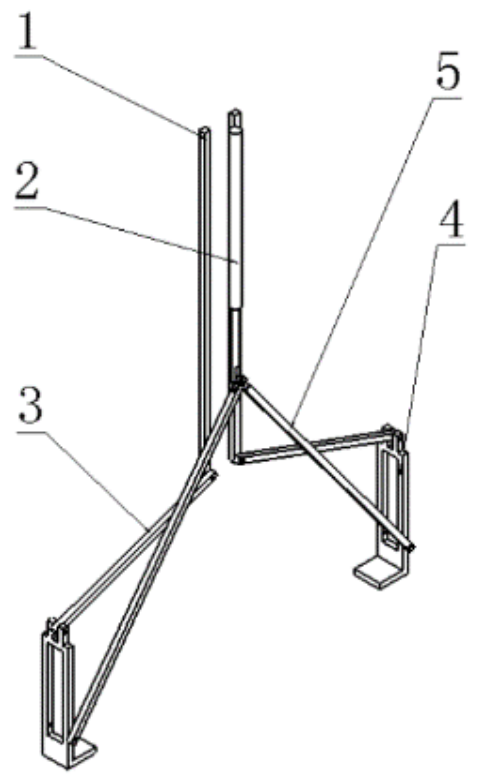

1. Vertical fixing rod 2. Electric putter 3. Horizontal fixing rod 4. Gripper 5. Connecting rod

Fig. 2 Palletizing manipulator

\subsection{Kinematics analysis of palletizing manipulator}

Determining the gripping position of the gripper and the minimum and maximum opening degrees that do not interfere with the seedling tray when the gripper is opened is necessary to ensure that the gripper can firmly grasp the seedling tray and does not interfere with the outer side after the seedling tray is released. The reasonable stroke range of the electric push rod is analyzed according to the position change of the gripper.

\subsubsection{Determination of the grab position}

The position where the gripper grabs the seedling tray is set as the gripping position, and the corresponding position of the electric push rod end is labeled as the gripping point as shown in Fig. 3. When the gripper is in the gripping position, the $M N$ section should be in a horizontal position to ensure gripping stability as shown in Fig. 4.

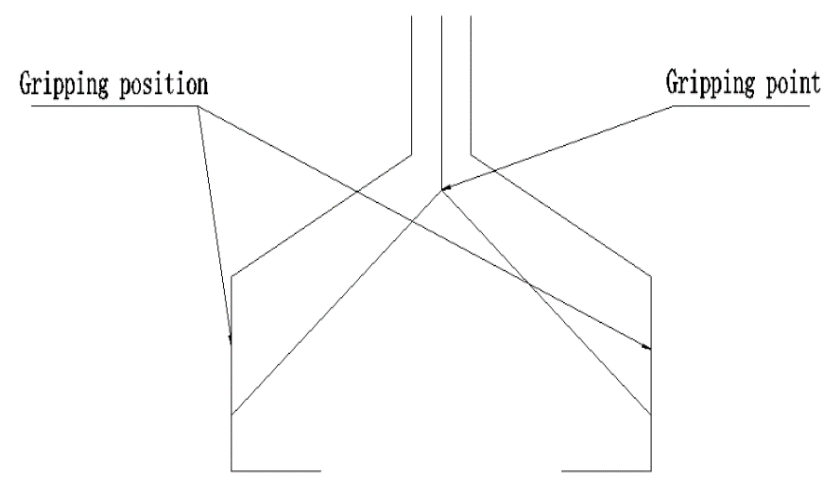

Fig. 3 Gripping position and point 


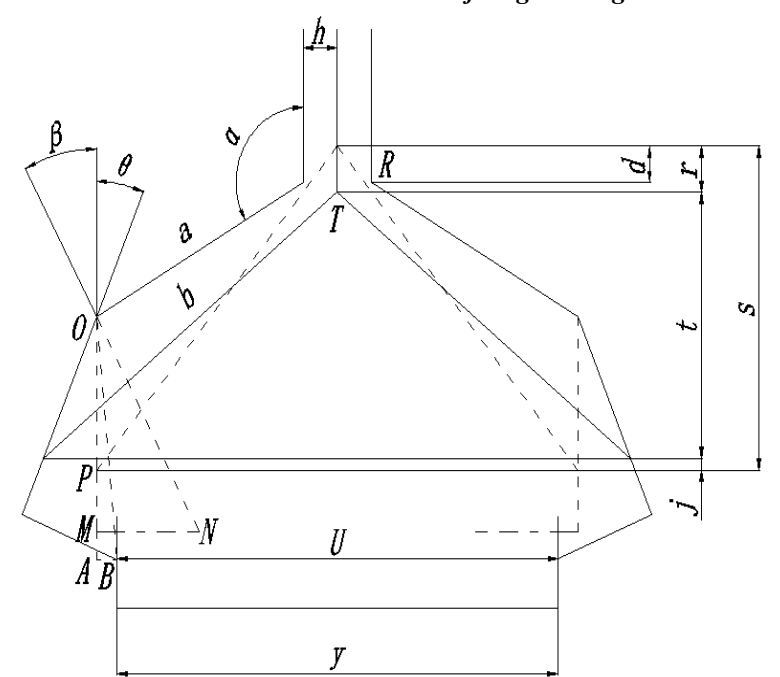

Note: $h$ represents distance of vertical fixing rod and electric putter, mm $\alpha$ represents angle between the vertical fixing rod and the horizontal fixing rod, $\left({ }^{\circ}\right) ; a$ represents length of horizontal fixing rod, $\mathrm{mm} ; b$ represents length of connecting rod; $\theta$ represents angle between grab position and minimum limit position, $\left(^{\circ}\right)$; $O P$ represents center distance of two hinge joint of gripper, $\mathrm{mm} ; M N$ represents length of grab flat, $\mathrm{mm}$; point $A$ represents pedal; point $B$ represents the point of contact between gripper and seeding tray when gripper is at minimum limit position; $y$ represents length of the seedling plate, $\mathrm{mm} ; j$ represents displacement of $P$ point in the vertical direction, $\mathrm{mm}$; point $T$ represents the hinge point of the electric push rod and the connecting rod, $\mathrm{mm} ; t$ represents the vertical distance between the point $P$ and $T$ point when gripper is at minimum limit position, $\mathrm{mm} ; s$ represents the vertical distance between the point $P$ and $T$ point when the gripper is at the grip of the position; $r$ represents the distance between the grabbing point and the minimum limit point, $\mathrm{mm}$; point $L$ represents the hinge point of the vertical fixed rod and the horizontal fixed rod; $d$ represents the vertical distance between the grabbing point and the $L$ point, $\mathrm{mm}$.

Fig. 4 Minimum limit point

According to the analysis in Fig. 4, $d$ is a definite value after $\alpha, a, b, O P$ and $h$ are determined. The position of gripping point can be determined by $d$ value.

It can be known from geometric relations:

$d=\left|s-a \sin \left(\alpha-90^{\circ}\right)-O P\right|$

$s=\sqrt{b^{2}-\left(\frac{y}{2}+\left(a+\frac{h}{\sin \alpha}\right) \sin \alpha-\frac{y}{2}\right)^{2}}$

Simplifying Equation (2) can obtain Equation (3).

$s=\sqrt{b^{2}-(a \sin \alpha+h)^{2}}$

Equation (4) is given by combining Equations (3) and (1).

$d=\left|\sqrt{b^{2}-(a \sin \alpha+h)^{2}}-a \sin \left(\alpha-90^{\circ}\right)-O P\right|$

Simplifying Equation (4) can obtain Equation (5).

$d=\left|\sqrt{b^{2}-(a \sin \alpha+h)^{2}}+a \cos \alpha-O P\right|$

\subsubsection{Determination of the minimum limit position}

When the gripper releases the seedling tray, the reasonable angle at which the gripper is opened and the corresponding extension distance for the electric pushrod must be determined to ensure that the hand gripper does not interfere with the outer side of the seedling tray's handle during the upward movement. When the interference does not happen, the position of the gripper is the minimum limit position, and the position of the corresponding end of the electric push rod is the minimum limit point. $\theta$ is the angle at which the gripper rotates from the gripping position to the minimum limit position, and $r$ is the distance of the electric push rod from the gripping point to the minimum limit point. $r$ is related to $a, b, h, \theta, \alpha, O P$ and other parameters, $\theta$ is unknown; and $\theta$ is related to $h, \alpha, O P, P M, M N$, and other parameters. Therefore, the relationship of $\theta$ with $h, \alpha, O P$, $P M$, and $M N$ should be first determined, followed by the relationship among $r$ and $a, b, h, \theta, \alpha, O P$, and other parameters. The expression of $r$ can be determined finally by bringing $\theta$ into $r$.

According to Fig. 4, the derivation process for $\theta$ and $r$ is as follows.

First, points $O$ and $N$ are connected. Second, line $O M$ is extended to point $A$. Then, vertical line $A B$ is drawn by passing $A$ point, and $B$ point is pedal. Finally, points $O$ and $B$ are connected. A relationship exists when the gripper is in the gripping position:

$$
A B=O B \cdot \sin (\beta-\theta)
$$

Simultaneously:

$$
A B=a \sin \alpha-\left(\frac{y}{2}-h\right)
$$

Equation (8) is obtained by merging Equations (6) and (7).

$$
a \sin \alpha-\left(\frac{y}{2}-h\right)=O B \cdot \sin (\beta-\theta)
$$

Simultaneously:

$\tan \beta=\frac{M N}{O M}$

$\beta=\arctan \frac{M N}{O M}$

Equation (11) is obtained by taking Equation (10) into Equation (8).

$$
\theta=\arctan \frac{M N}{O P+P M}-\arcsin \frac{a \sin \alpha-\frac{y}{2}+h}{\sqrt{(O P+P M)^{2}+M N^{2}}}
$$

Equation (12) can be obtained from the geometric relationship in Fig. (4).

$$
r=s-t-j
$$

Simultaneously:

$s=\sqrt{b^{2}-(a \sin \alpha+h)^{2}}$ 
$t=\sqrt{b^{2}-(a \sin \alpha+h+O P \cdot \sin \theta)^{2}}$

$j=O P \cdot(1-\cos \theta)$

Taking Equations (13)-(15) into (12) can obtain Equation (16).

$$
\begin{aligned}
& r=\sqrt{b^{2}-(a \sin \alpha+h)^{2}}-\sqrt{b^{2}-(a \sin \alpha+h+O P \cdot \sin \theta)^{2}} \\
& -O P \cdot(1-\cos \theta)
\end{aligned}
$$

Equation (17) is given by taking Equations (11) into (16).

$$
\begin{array}{r}
r=\sqrt{b^{2}-(a \sin \alpha+h)^{2}}-\sqrt{b^{2}-\left(a \sin \alpha+h+O P \cdot \sin \left(\arctan \frac{M N}{O P+P M}-\arcsin \frac{a \sin \alpha-\frac{y}{2}+h}{\sqrt{(O P+P M)^{2}+M N^{2}}}\right)\right)^{2}} \\
-O P \cdot\left(1-\cos \left(\arctan \frac{M N}{O P+P M}-\arcsin \frac{a \sin \alpha-\frac{y}{2}+h}{\sqrt{(O P+P M)^{2}+M N^{2}}}\right)\right)
\end{array}
$$

From the initial conditions of the derivation and the expressions of $\theta$ and $r, \theta$ is only established when the gripper is at the minimum limit position. $r$ is a general formula and is not limited by the relative positions of the gripper and the seedling tray.

\subsubsection{Determination of the maximum limit position}

The stacking manipulator is a symmetrical structure with the electric push rod as the axis of symmetry. For the simplified analysis, only the left part of the electric push rod is analyzed. The left part is a plane four-bar linkage. If the frictional force is in the motion pair, then the influence of gravity and the inertial force of the component are not considered. The link axis is collinear with the $O P$ connection in the position shown in Fig. 5, that is, the transmission angle is $0^{\circ}$. At this time, the force of the electric push rod acts on the gripper through the connecting rod passing through the center of the rotation center of the gripper $(O)$, which is the dead center position of the palletizing robot [31] Owing to the small mass of the components of the stacking manipulator, the dead center position cannot smoothly pass through the inertia. Therefore, when the manipulator is in the dead position, the position of the gripper is taken as the maximum limit position, and the position of the corresponding electric push rod end is taken as the maximum limit point. $\lambda$ is the angle at which the gripper rotates from the gripping position to the maximum limit position, and $\mathrm{k}$ is the distance from the gripping point to the maximum limit point of the electric push rod. The previous analysis indicates that $\theta$ does not hold when the gripper is at the maximum limit position, and the $\lambda$ formula needs to be re-derived.

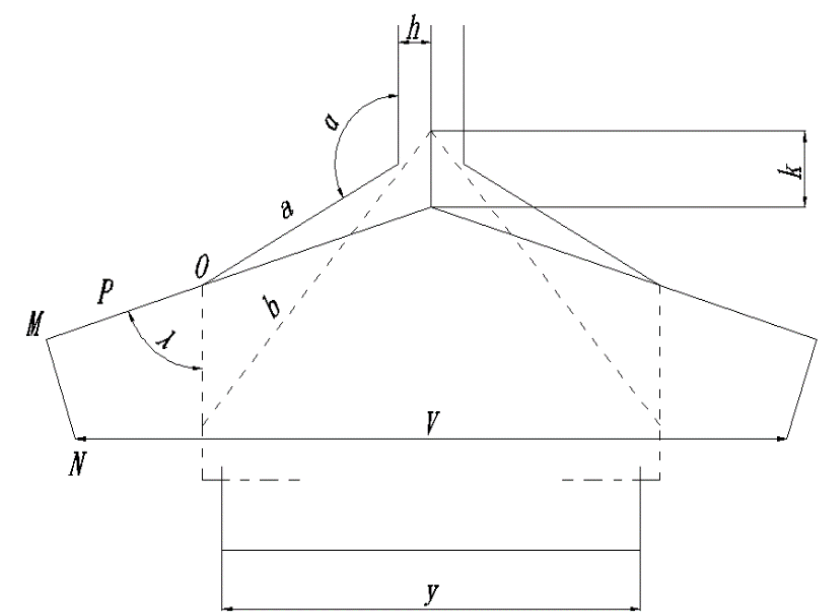

Note: $\lambda$ represents the angle between the grab position and the maximum limit position, $\left(^{\circ}\right) ; k$ represents the distance between the grabbing point and the maximum limit point.

Fig. 5 Maximum limit position and point

Equation (18) is given based on the analysis in Figure 5.

$$
\begin{aligned}
& \sin \lambda=\frac{a \sin \alpha+h}{b-O P} \\
& \lambda=\arcsin \frac{a \sin \alpha+h}{b-O P}
\end{aligned}
$$

Equation (20) is obtained by taking Equation (19) into Equation (17). (Note: replace $\theta$ with $\lambda$ in Equation (17)).

$$
k=\sqrt{b^{2}-(a \sin \alpha+h)^{2}}-\sqrt{b^{2}-\left(a \sin \alpha+h+O P \cdot \sin \left(\arcsin \frac{a \sin \alpha+h}{b-O P}\right)\right)^{2}}-O P \cdot\left(1-\cos \left(\arcsin \frac{a \sin \alpha+h}{b-O P}\right)\right)
$$

Simultaneously:

$k=\sqrt{b^{2}-(a \sin \alpha+h)^{2}}-\sqrt{b^{2}-\left(a \sin \alpha+h+O P \cdot \frac{a \sin \alpha+h}{b-O P}\right)^{2}}-O P \cdot\left(1-\cos \left(\arcsin \frac{a \sin \alpha+h}{b-O P}\right)\right)$

In conclusion, $\eta$ should satisfy the relation to open the gripper without interfering with the outer surface of the seedling tray and to ensure the stroke of the electric putter is small. The seedling tray is standardized as shown in Fig. 6. Its parameters are given in Table 1 . 


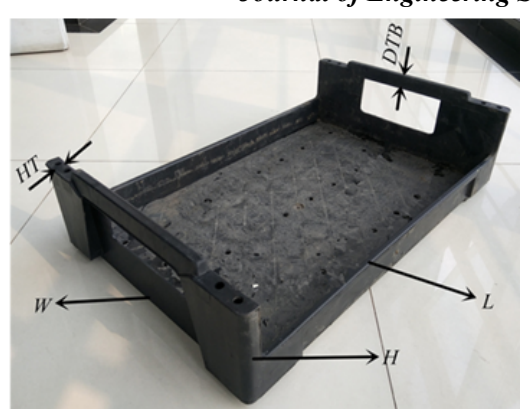

Fig. 6 Seedling tray

Table 1 Parameter of seedling tray

\begin{tabular}{l|l}
\hline Parameter & Size \\
\hline Length $(L), \mathrm{mm}$ & 688 \\
Width $(W), \mathrm{mm}$ & 440 \\
Height $(H), \mathrm{mm}$ & 180 \\
Handle thickness $(H T), \mathrm{mm}$ & 24 \\
Distance between top and bottom & 42.92 \\
of handle $(D T B), \mathrm{mm}$ & \\
\hline
\end{tabular}

The restrictive conditions of palletizing manipulator are as follows.

$$
\left\{\begin{array}{l}
P M \geq 42.92, M N \geq 24, y=688 \\
h \geq 0, O P \geq 0 \\
90^{\circ} \leq \alpha<180^{\circ} \\
h+a \sin \alpha \geq \frac{y}{2} \\
b>h+a \sin \alpha
\end{array}\right.
$$

The combined seedling tray parameters in Table 1 and the restrictive conditions could be used to obtain the partial parameter of the manipulator. However, the values of $b$ and $O P$ could not be directly determined and could only be calculated through the statics analysis of the palletizing manipulator.

\subsection{Palletizing manipulator's statics analysis}

The mass of the seedling tray after filling the substrate is 13 $\mathrm{kg}$. The selected electric push rod has a rated thrust of $75 \mathrm{~N}$ and a self-locking force of $150 \mathrm{~N}$ to meet the requirements of grab handling. The material selected for each rod is standard hard aluminum alloy, grade 2A11, and its yield limit $\delta_{b}=375 \mathrm{Mpa}$ [29]. Fig. 7 shows the force of the palletizer.

The statics equilibrium equation is as follows:

$\sum F_{x}=0, F_{o x}+F_{p x}=0$

$\sum F_{y}=0, F_{o y}+F_{p y}-\frac{m \mathrm{~g}}{2}=0$

$\sum M_{o}=0, F_{p x} \cdot O P-\frac{M D \cdot m \mathrm{~g}}{2}=0$

$F=-2 F_{p y}$

$F_{p}=\sqrt{F_{p x}^{2}+F_{p y}^{2}}$

$$
\begin{aligned}
& F_{c r}=\frac{\pi^{2} E I}{b^{2}} \\
& n_{s t}=\frac{F_{c r}}{F_{p}} \\
& \tan \varepsilon=\frac{F_{p y}}{F_{p x}} \\
& \cos \varepsilon=\frac{0.349}{b}
\end{aligned}
$$

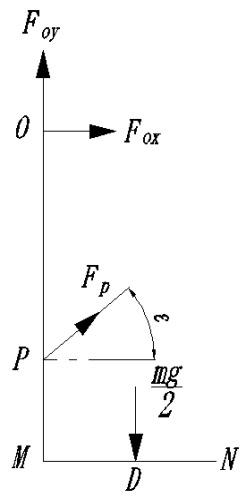

Fig. 7 Force analysis of gripper

Note: $m$ represents the quality of the seedling plate, $\mathrm{kg}$; $M D$ represents the distance between the equivalent stress point and the inner side of the distance, $\mathrm{mm} ; F_{c r}$ represents critical pressure, N; $E$ represents the modulus of elasticity of a connecting rod, GPa; $I$ represents the moment of inertia of the cross-section of a connecting rod, $\mathrm{m}^{4} ; F$ represents the force of an electric push rod, $\mathrm{N} ; n_{s t}$ represents stable safety factor; $\varepsilon$ represents the angle between the connecting rod and the gripper, $\left({ }^{\circ}\right)$.

The known conditions of static balance equations are as follows:

$m=13 \mathrm{~kg}, \mathrm{~g}=9.8 \mathrm{~N} / \mathrm{kg}, F=150 \mathrm{~N}, M D=17 \mathrm{~mm}, n_{s t}=5$

$E=70 \mathrm{GPa}, I=\frac{10 \times 10^{-3} \times\left(6 \times 10^{-3}\right)}{12} \mathrm{~m}^{4}=1.8 \times 10^{-4} \mathrm{~m}^{4}$

The result of static equilibrium Equations (22)-(30) is as follows:

$O P=13.22 \mathrm{~mm}, \quad b=473.27 \mathrm{~mm}$

Equation (27) is the Euler formula for the hinged pressure rods at both ends, but only the large flexible pressure rods satisfy the Euler's equation. Therefore, proving that the connecting rod is a large flexible pressure rod is necessary, and the process for proof is as follows:

$$
\begin{aligned}
& i=\sqrt{\frac{I}{Q}} \\
& \tau=\frac{b}{i}
\end{aligned}
$$




$$
\tau_{1}=\pi \sqrt{\frac{E}{\sigma_{p}}}
$$

The known conditions are as follows:

$$
\begin{aligned}
& b=473.27 \mathrm{~mm} \\
& I=1.8 \times 10^{-10} \mathrm{~m}^{4} \\
& E=70 \mathrm{GPa} \\
& \delta_{b}=375 \mathrm{MPa} \\
& Q=6 \times 10^{-5} \mathrm{~m}^{2} \\
& \sigma_{p}=2.9607+0.3692 \sigma_{b}
\end{aligned}
$$

Note: $i$ represents the inertial radius of the cross-section of the connecting rod, $\mathrm{m} ; Q$ represents cross-section area of connecting rod, $\mathrm{m}^{2} ; \tau$ represents the flexibility of the connecting rod; $\sigma_{p}$ represents ratio limit of connecting rod, $\mathrm{MPa} ; \sigma_{b}$ represents the yield limit of the connecting rod, $\mathrm{MPa}$.

The result for Equations (31)-(33) is as follows:

$$
\tau=273.23, \tau_{1}=69.90
$$

This finding indicates that when $b \geq 473.23$, the connecting rod is a large flexible rod, namely, the above static equilibrium equations are still true.

\section{Result Analysis and Discussion}

\subsection{Determination of key parameters}

According to the above analysis, the optimal values of $O P$ and $b$ are 13.22 and $473.27 \mathrm{~mm}$, respectively. However, the unloading palletizing device must be used together with the square seedling tray planter to ensure the stability of the whole machine. In addition, the height of the unloading palletizing device should not exceed the height of square seedling tray planter. Under this limit condition, the unloading palletizing device can stack up to 7 trays at most. When the 7 seedling trays are palletized, the $Z$ direction slider of the unloading palletizing device must be at the upper limit switch, and the distance between gripping surface of the gripper and the ground is greater than or equal to the distance between the gripping surface of seedling tray at the top of the plate and the ground. When the $Z$ direction slider of the palletizing device is at the lower limit switch, the distance between the gripping surface of the gripper and the ground is less than or equal to the distance between the gripping surface of seedling tray at the under of the plate and the ground. Combining the above analysis, we could obtain two conditions of $O P$.

$$
\left\{\begin{array}{l}
1263.40 \mathrm{~mm}-O P-P M \geq 1037.08 \mathrm{~mm} \\
1461.75 \mathrm{~mm}-1120.51 \mathrm{~mm}-O P-P M \leq 137.08 \mathrm{~mm}
\end{array}\right.
$$

The scope of the $O P$ is:

$$
161.16 \mathrm{~mm} \leq O P \leq 183.32 \mathrm{~mm}
$$

The theoretical value of $O P$ obtained in section 3 is $13.22 \mathrm{~mm}$, which does not meet the requirements for an unloading palletizing device that stacks up to 7 seedling trays. Therefore, the value of $O P$ must be appropriately increased. Structural analysis of palletizing manipulator shows that increasing the $O P$ value also increases the $r$ value, thereby prolong the time that electric putter moves from the grasping point to the minimum limit point and reducing the working efficiency of the palletizing manipulator. Increasing the value of $b$ decreases the value of $r$, thereby reducing the time. Therefore, values $O P$ and $b$ should be increased to reduce the $r$ value and ensure the working efficiency of the palletizing manipulator.

Table 2 Numerical changes of $O P$ and $b$

\begin{tabular}{l|l|l|l|l|l}
\hline $\boldsymbol{O P} / \mathbf{m m}$ & $\boldsymbol{b} / \mathbf{m m}$ & $\boldsymbol{F}_{\boldsymbol{p}} / \mathbf{N}$ & $\boldsymbol{F}_{\boldsymbol{c}} \boldsymbol{N} \mathbf{N}$ & $\boldsymbol{n}_{\boldsymbol{s} \boldsymbol{t}}$ & $\boldsymbol{r} / \mathbf{m m}$ \\
\hline 13.22 & 473.27 & 111.06 & 555.20 & 5.00 & 6.31 \\
33.22 & 483.27 & 45.15 & 532.46 & 11.79 & 13.84 \\
53.22 & 493.27 & 28.77 & 511.09 & 17.76 & 18.98 \\
73.22 & 503.27 & 21.33 & 490.98 & 23.02 & 22.38 \\
93.22 & 513.27 & 17.09 & 472.04 & 27.62 & 24.58 \\
113.22 & 523.27 & 14.33 & 454.17 & 31.69 & 25.98 \\
133.22 & 533.27 & 12.42 & 437.30 & 35.21 & 26.85 \\
153.22 & 543.27 & 11.00 & 421.35 & 38.30 & 27.35 \\
173.22 & 553.27 & 9.91 & 406.25 & 40.99 & 27.59 \\
193.22 & 563.27 & 9.03 & 391.96 & 43.41 & 27.65 \\
213.22 & 573.27 & 8.34 & 378.40 & 45.37 & 27.59 \\
233.22 & 583.27 & 7.75 & 365.54 & 47.17 & 27.43 \\
253.22 & 593.27 & 7.27 & 353.32 & 48.60 & 27.21 \\
273.22 & 603.27 & 6.84 & 341.70 & 49.96 & 26.95 \\
293.22 & 613.27 & 6.48 & 330.65 & 51.03 & 26.65 \\
\hline
\end{tabular}

Basing on the above analysis and the data in Table 2, we use 173.22 and $553.27 \mathrm{~mm}$ as the optimal values for $O P$ and $b$, respectively. From the response surface in Figure 8 , the value of $r$ increases with increasing $O P$ and decreases with increasing $b$. This trend verifies the accuracy of the structural analysis for the palletizing manipulator.

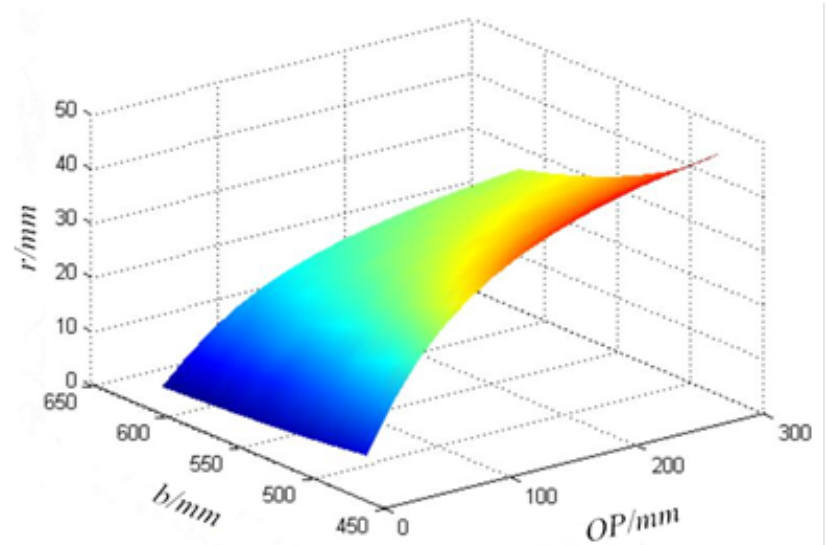

Fig. 8 Influence on $r$ value with the changes of values $b$ and $O P$

\subsection{Simulation analysis}

On the basis of the parameter values obtained above, the 3D model of the palletizing manipulator was rebuilt by SolidWorks and introduced into Adams for simulation. The value of $d$ is determined when the parameters of the palletizing manipulator are known. Hence, the simulation of $d$ value is placed in the statics simulation. Kinematics simulation is conducted under the conditions that the grippers are at the minimum and maximum limit positions.

(1) Simulation when the gripper is at minimum limit position: the obtained parameters are inputted into Equation (11) to obtain $\theta=11.7296^{\circ}$. The value of $\theta$ is inputted into Adams to obtain the values of $r$ and $U$, which are 27.59 and $688 \mathrm{~mm}$, respectively.

(2) Simulation when the gripper is at the maximum limit position: the obtained parameters are inputted into Equation (19) to obtain $\lambda=66.7^{\circ}$. The value of $\lambda$ is inputted into Adams to obtain the values of $k$ and $V$, which are 105.63 and $1055.52 \mathrm{~mm}$, respectively. 


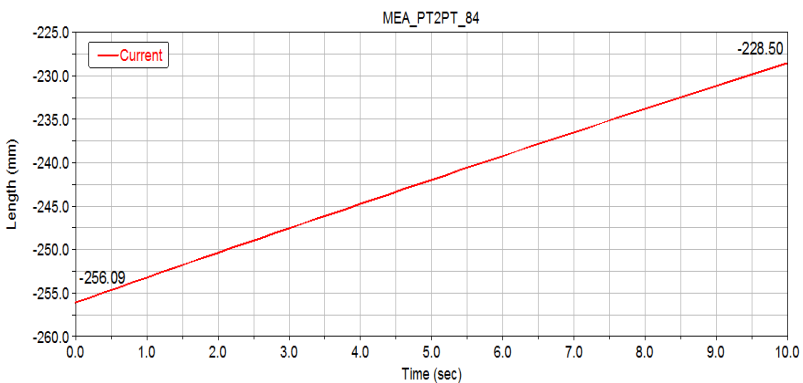

(a) Simulation of $r$

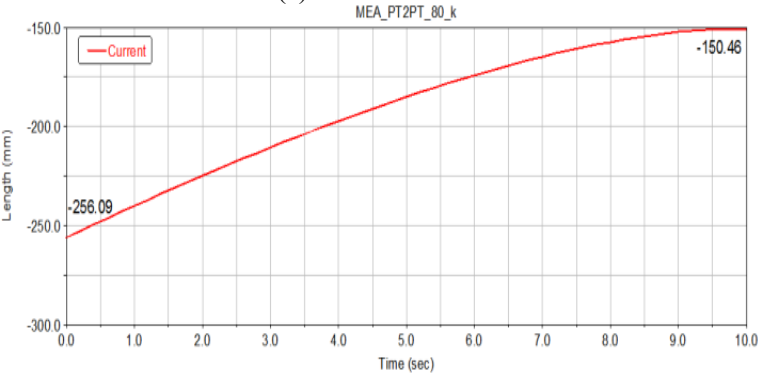

(b) Simulation of $k$ MEA PT2PT 80

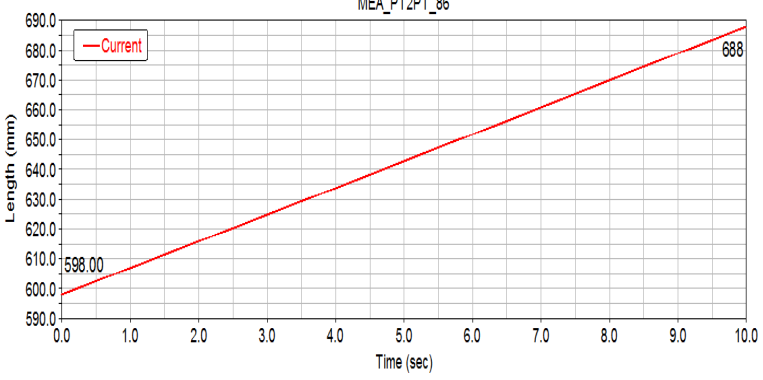

(c) Simulation of $U$

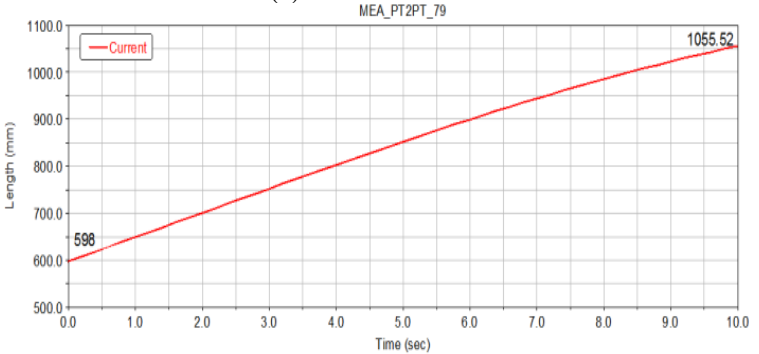

(d) Simulation of $V$

Fig. 9 Kinematics simulation

Statics simulation: the values of $d$, Fox, Foy, Fpx, Fpy, and $F$ are $256.1 \mathrm{~mm}, 6.25 \mathrm{~N}, 56.01 \mathrm{~N}, 6.25 \mathrm{~N}, 7.69 \mathrm{~N}$, and $15.38 \mathrm{~N}$, respectively, as shown in Fig. 10(a) to 10(f).

Analysis in Table 3 shows that the values of theoretical calculation coincide with the simulation values. Hence, the simulation results verify the accuracy of the modeling and theoretical analysis.

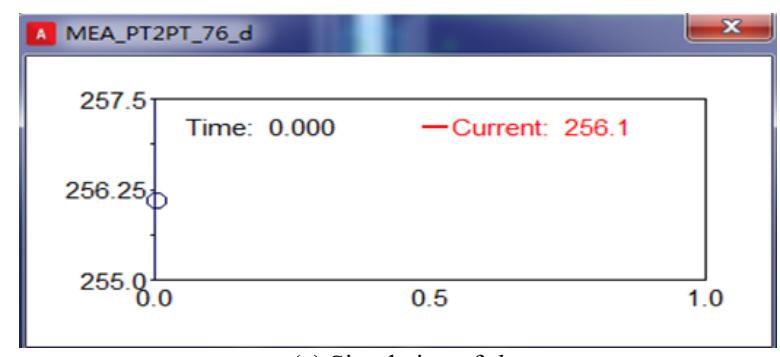

(a) Simulation of $d$

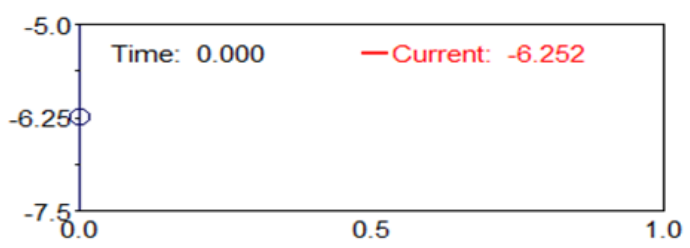

(b) Simulation of $F_{o x}$
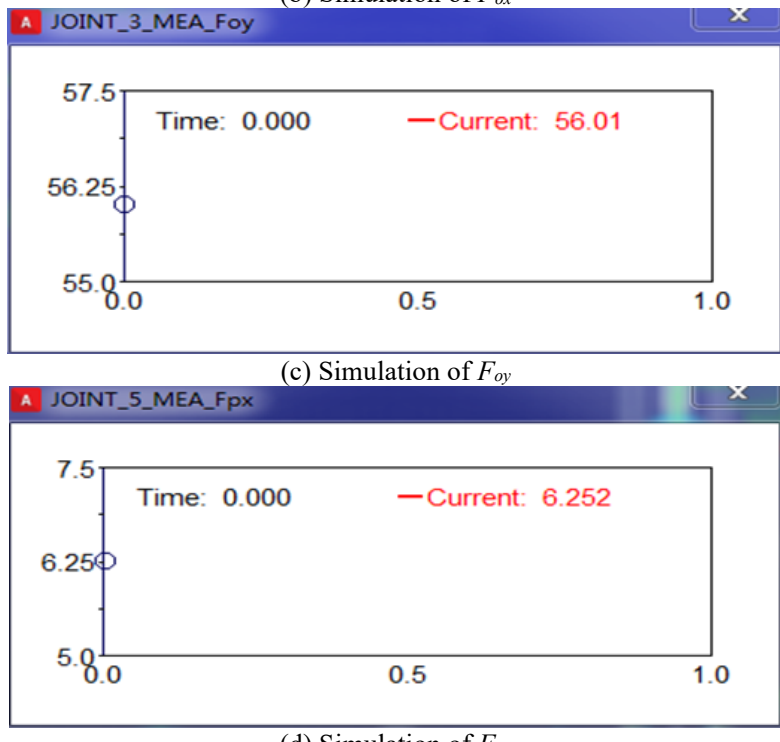

Fig. 10 Statics simulation

(d) Simulation of $F_{p x}$

Table 3. Comparison between theoretical and simulation values

\begin{tabular}{c|c|c|c|c}
\hline Parameter & $\begin{array}{l}\text { Theoretical } \\
\text { calculation } \\
\text { value }\end{array}$ & $\begin{array}{l}\text { Simulatio } \\
\text { n value }\end{array}$ & Deviation & $\begin{array}{l}\text { Degree of the } \\
\text { coincidence/\% }\end{array}$ \\
\hline$d / \mathrm{mm}$ & 256.09 & 256.10 & 0.01 & 99.996 \\
\hline$r / \mathrm{mm}$ & 27.59 & 27.59 & 0 & 100 \\
\hline$U / \mathrm{mm}$ & 688.01 & 688.00 & 0.01 & 99.999 \\
\hline$k_{/ \mathrm{mm}}$ & 105.64 & 105.63 & 0.01 & 99.991 \\
\hline$V_{/ \mathrm{mm}}$ & 1055.54 & 1055.52 & 0.02 & 99.998 \\
\hline$F_{o x} / \mathrm{N}$ & 6.25 & 6.25 & 0 & 100 \\
\hline$F_{o y} / \mathrm{N}$ & 56.01 & 56.01 & 0 & 100 \\
\hline$F_{p x} / \mathrm{N}$ & 6.25 & 6.25 & 0 & 100 \\
\hline$F_{p \mathrm{y}} / \mathrm{N}$ & 7.69 & 7.69 & 0 & 100 \\
\hline$F_{/ \mathrm{N}}$ & 15.38 & 15.38 & 0 & 100 \\
\hline
\end{tabular}

\subsection{Effect of seedling speed}

The above simulation process is based on the system under a static state but without consideration on the vibration of the whole device and the influence of the inertia of the robot arm on the gripping of the end manipulator. The working process of the seedling tray handling and palletizing can be divided into three stages, namely, the grasping of the seedling tray, the lateral handling, and the palletizing of the seedling tray. The lateral handling of the seedling tray has the largest stroke, the fastest running speed for the seedling tray handling device, and the greatest effect on the stability of the device. Hence, the influence on the gripping of the robot is the greatest. With the change of seedling speed, the impact force of the three processes of acceleration, uniform speed, and deceleration in lateral transportation changes 
correspondingly, thus altering the magnitude of vibration and positioning error [32]. A high seedling speed produces great vibration and error. Therefore, in practical applications, the anti-shake and shock-proof design of the device should be considered, and the motion control theory should be added to increase the running stability of the manipulator and reduce the influence of the seedling speed on the gripping end of the manipulator.

\section{Conclusions}

This study designed an automatic unloading and palletizing device to solve high labor intensity and low efficiency in manual operation. Through the analysis of the action flow of the manual operation, the structural and dynamic analysis of the manipulator was conducted, the mathematical model was established, and the simulation was verified. Finally, the following conclusions were obtained:

(1) The reasonable stroke range of the electric push rod is $27.6-105.6 \mathrm{~mm}$, and the opening/closing angle of the claw is $11.7^{\circ}-66.7^{\circ}$, which is determined by the minimum and maximum limit positions of the manipulator.

(2) From the analysis of the structure of the manipulator and the distance from the grab point to the minimum limit point, the response surface map shows that the center distance $O P$ of the two hinge points of the claw will increase, and the length of the link $b, r$ will decrease slightly. Therefore, the $O P$ and $b$ should be simultaneously increased to slow down the increase in the $r$ value and ensure the working efficiency of the robot.

(3) SolidWorks is used to perform the $3 \mathrm{D}$ modeling verification of the palletizing robot with known parameters and import it into Adams for kinematics and statics simulation. The theoretical calculation results are consistent with the simulation results, thus verifying the correctness of theoretical analysis and parameter optimization.

This study combines theoretical calculation and simulation analysis to design and optimize a square substrate seedling machine palletizing robot. This work provides important reference to the prototype's manufacture and testing By analyzing the parameters of the seedling tray and the size of the manipulator and obtaining the values and ranges of each key parameter. Owing to the lack of prototype verification and actual field test data, the prototype test data will be combined with the simulation optimization of this model and further revised to make the structure of the square seedling machine palletizing robot reasonable, reliable, and highly efficient.

\section{Acknowledgments}

This work was supported by the National Key Research and Development Program of China (Grant Nos. 2017YFD0701500), the National Modern Agricultural Industry Technical System-Specialized Vegetable Production Mechanization Construction Special (Grant Nos. CARS-24-D-01), the Modern Agriculture Industry Technology System in Shandong Province (Grant Nos. SDAIT-05-11) and the Shandong Agricultural Major Application Technology Innovation.

This is an Open Access article distributed under the terms of the Creative Commons Attribution License

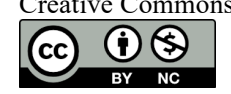

\section{References}

1. Zhao, Z., Wang, J., Liu, L., "Advance research of tray precision sowing equipment". Journal of Agricultural Mechanization Research, 37 (8), 2015, pp. 1-5+25.

2. $\mathrm{Wu}, \mathrm{G}$., "Advances of research on precision seeding equipment with plug trays in greenhouse". Northern Horticulture, 34(15), 2010, pp.37-41.

3. Chen, D., "Summary of foreign and domestic vegetable corps plug transplants production". Chinese Vegetables, S1, 2000, pp.9-13.

4. Liu, Z., Hou, J., Xin, J., "Design and test of intelligent facility seedling raising machine". Transactions of The Chinese Society of Agricultural Machinery, 47(S1), 2016, pp.136-142.

5. Kim, D.E., Chang, Y.S., Kim, S.H., Lee, G.I., "Development of vacuum nozzle seeder for cucurbitaceous seeds (I) - design factors for vacuum seeding large sized seeds". Journal of the Korean Society for Agricultural Machinery, 28(6), 2003, pp.525-530.

6. Jiang, K., Zhang, Q., Wang, X., "Design and test on self-cleaning seeding head for mechanism suction". Transactions of the Chinese Society of Agricultural Engineering, 29(20), 2013, pp.18-23.

7. Gaikwad, B.B. Sirohi, N.P.S., "Design of a low-cost pneumatic seeder for nursery trays". Biosystems Engineering, 99, 2008, pp.322-329.

8. Singh, R.C., Singh, G., Saraswat, D.C., "Optimization of design and operational parameters of a pneumatic seed metering device for planting cotton seeds". Biosystems Engineering, 92(4), 2005, pp.429-438.

9. Guarella, P., Pellerano, A., Pascuzzi, S., "Experimental and theoretical performance of a vacuum seeder nozzle for vegetable seeds". Journal of Agriculture Engineering Research, 64, 1996, pp.29-36.

10. Yamada, H., Shimizu, S., Kobayashi, K., Fujii, M., Sasaya, S., "Development of aligning seeder for big and elliptical seeds". Journal of the Japanese Society of Agricultural Machinery, 67(5), 2005, pp.109-116.
11. Yu, Y., Wu, P., Feng, Q., "Situation and strategy of automatic seedling machine in China". Journal of Agricultural Mechanization Research, 39(6), 2017, pp.1-6.

12. Zhao, Y. "Studies on the intelligent management and Comprehensive Evaluation of Industrialized Vegetable Seedling". Doctoral Dissertation of Jilin University, China, 2011, pp.24-30.

13. Liu, Y., Li, Y., Xu, X., "Study on Development of Plug Seedling Planter and Technology". Agricultural Science \& Technology and Equipment, 6, 2014, pp.34-36.

14. Chiu, Y.C., Chu, Y.H., Wu, G.J., "Development of an automatic tray-discharging system for rice seedlings". Engineering in agriculture, environment and food, 6(1), 2013, pp.27-33.

15. Chiu, Y.C., Wu, G.J., Chen, C.H., "Development of a generatorpowered self-propelled automatic tray loading/unloading machine for use in rice nurseries". Engineering in Agriculture, Environment and Food, 12(2), 2019, pp.256-63.

16. Xiao, Z., Wu, G., Zheng, Y., Tan, Y., Lyu, H., "Design and Test of Automatic Feed and Send System for Combined Trays Seedlings Transplanter”. In: 2017 ASABE Annual International Meeting, St. Joseph, USA: American Society of Agricultural and Biological Engineers, 2017, pp.1-9.

17. Jin, X., Li, M., Li, D., Ji, J., Pang, J., Wang, J., Peng, L., "Development of automatic conveying system for vegetable seedlings". EURASIP Journal on Wireless Communications and Networking. (1), 2018, pp.178.

18. Rosli, M.F., Mahadi, M.R., Misri, M.A., Wayayok, A., "Initial design of an automated system for paddy seedling placement in a germination tray". Jurnal Teknologi. 78 (1-2), 2016, pp.119-123.

19. Han, L., Mao, H., Hu, J., Tian, K., "Development of a doorframetyped swinging seedling pick-up device for automatic field transplantation". Spanish journal of agricultural research. (2), 2015, pp.13.

20. Choi, W. C., Kim, D. C., Ryu, I. H., Kim, K. U., "Development of a seedling pick-up device for vegetable transplanters". Transactions of the ASAE, 45(1), 2002, pp.13. 
Tianhua Li, Guangwei Liu, Yuhua Li, Yueshun Zhang, Jialin Hou, Guoying Shi and Kai Zhou/

\section{Journal of Engineering Science and Technology Review 12 (5) (2019) 157 - 166}

21. Ma, X., Tan, Y., Qi, L., Lu, F., Sun, G., Chen, G., “Automatic Tray Stacking Device for Hard and Soft Tray of Rice Precision Seeding for Nursing Seedlings Pipeline". Transactions of the Chinese Society for Agricultural Machinery,47(3), 2016, pp.29-36.

22. Ma, X., Tan, Y., Qi, L., Huang, G., Lu, F., Chen, L., "Design and test on pneumatic type automatic tray feeder of seeder for tray nursing seedings of rice". Transactions of the Chinese Society of Agricultural Engineering, 32(22), 2016, pp. 63-69. (in Chinese with English abstract)

23. Quan, L., Shen, J., Xi, D., Wang, H., Liu, L., "Motion Planning and Test of Robot for Seedling Tray Handling in Narrow Space". Transactions of the Chinese Society for Agricultural Machinery, 47(1), 2016, pp.51-59.

24. Li, S., Cao, W., Tang, H., Zhao, H., Liu, J., Li, H., “A Study on Dynamic of Transmission Mechanism of Seedling Tray", Journal of Agricultural Mechanization Research, 39(12), 2017, pp.15$19+25$.

25. Wei, T., Zhang, S., "Design of $2 \mathrm{YCB}$ pneumatic tobacco tray seeder". Journal of Chinese Agricultural Mechanization, 36(05), 2015, pp.5-8.

26. Ren, L., Cao, W., Ma, R., Wang, N., "Design of seedling tray conveying control system based on lab view". Journal of Agricultural Mechanization Research, 41(09), 2019, pp.104-109.
27. Jin, X., Du, Xi., Yang, C., Ji, J., Dong, Z., Yan, H., "Design and Experiment on Automatic Transporting Mechanism for Vegetable Potted Seedlings". Transactions of the Chinese Society for Agricultural Machinery, 47(7), 2016, pp.103-111.

28. Chiba R., Arai T., Ueyama T., Ogata T., Ota J., "Working environment design for effective palletizing with a 6-DOF manipulator". International Journal of Advanced Robotic Systems, 13(2), 2016, pp.68.

29. Nakamoto, H., Eto, H., Sonoura, T., Tanaka, J., Ogawa, A., "Highspeed and compact depalletizing robot capable of handling packages stacked complicatedly". In: 2016 IEEE/RSJ International Conference on Intelligent Robots and Systems (IROS), Daejeon, Korea: IEEE, 2016, pp. 344-349.

30. Nguyen Q.C., Kim Y., Kwon H., "Optimization of layout and path planning of surgical robotic system". International Journal of Control, Automation and Systems, 15(1), 2017, pp.375-84.

31. García, E. L., Fontanet, J. V., Nebot, L. J., "Feasibility of motion laws for planar one degree of freedom linkage mechanisms at dead point configurations". Mechanical Systems and Signal Processing, 98, 2018, pp.834-851.

32. Li, T., Zhang, Y., Hou, J., Shi, G., Lou, W., Wu, Y., "Kinematics simulation analysis of seedling tray transport and palletizing device based on ADAMS". Journal of Chinese Agricultural Mechanization, 39(10), 2018, pp.01-06. 